\title{
TITLE:
}

\section{Theoretical study on ionization process in aqueous solution.}

\section{$\operatorname{AUTHOR}(\mathrm{S}):$}

lida, Kenji; Sato, Hirofumi

\section{CITATION:}

lida, Kenji ... [et al]. Theoretical study on ionization process in aqueous solution.. The Journal of chemical physics 2012, 136(14): 144510.

ISSUE DATE:

2012-04-14

URL:

http://hdl.handle.net/2433/155462

RIGHT:

(C) 2012 American Institute of Physics. 


\section{AIP $\begin{gathered}\text { molowanat } \\ \text { chemical Physics }\end{gathered}$}

\section{Theoretical study on ionization process in aqueous solution}

Kenji lida and Hirofumi Sato

Citation: J. Chem. Phys. 136, 144510 (2012); doi: 10.1063/1.3700225

View online: http://dx.doi.org/10.1063/1.3700225

View Table of Contents: http://jcp.aip.org/resource/1/JCPSA6/v136/i14

Published by the American Institute of Physics.

\section{Additional information on J. Chem. Phys.}

Journal Homepage: http://jcp.aip.org/

Journal Information: http://jcp.aip.org/about/about_the_journal

Top downloads: http://jcp.aip.org/features/most_downloaded

Information for Authors: http://jcp.aip.org/authors

\section{ADVERTISEMENT}

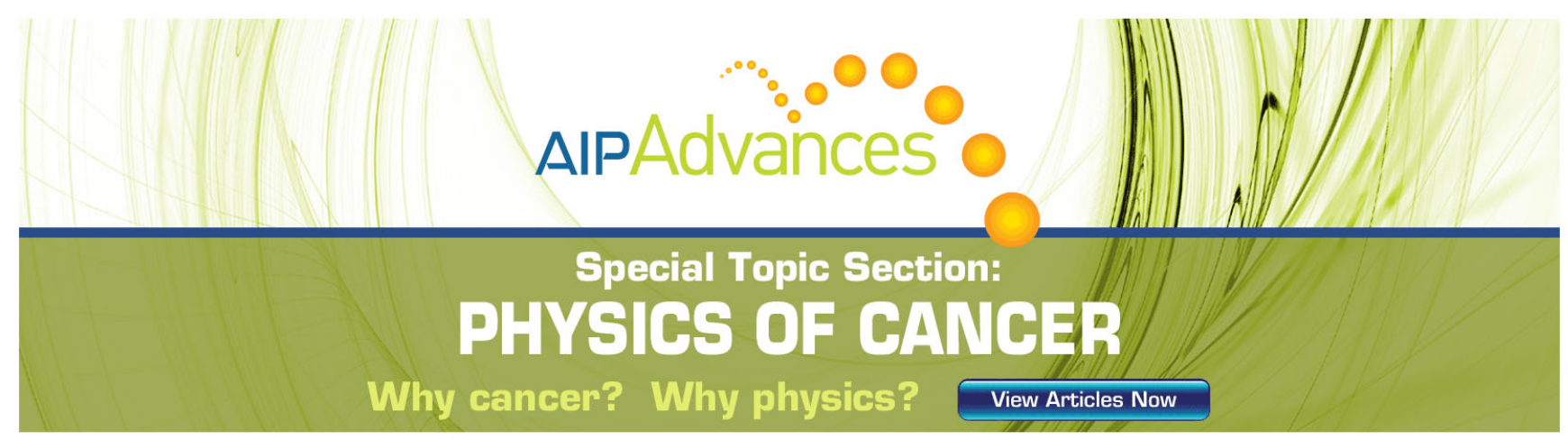




\title{
Theoretical study on ionization process in aqueous solution
}

\author{
Kenji lida and Hirofumi Sato ${ }^{a)}$ \\ Department of Molecular Engineering, Kyoto University, Kyoto 615-8510, Japan
}

(Received 30 January 2012; accepted 19 March 2012; published online 13 April 2012)

\begin{abstract}
Ionization potential (ionization energy) is a fundamental quantity characterizing electronic structure of a molecule. It is known that the energy in solution phase is significantly different from that in the gas phase. In this report, vertical and adiabatic ionization processes in aqueous solution are studied based on a hybrid method of quantum chemistry and statistical mechanics called reference interaction site model-SCF-spacial electron density distribution method. A role of solvation effect is elucidated through molecular level information, i.e., solvent distribution function around solute molecule. By utilizing the linear response regime, a simple expression to evaluate the spectral width from the distribution function is proposed and compared with experimental data. (C) 2012 American Institute of Physics. [http://dx.doi.org/10.1063/1.3700225]
\end{abstract}

\section{INTRODUCTION}

Ionization energy is a fundamental quantity characterizing electronic structure. It is classified in terms of the presence of relaxation process, namely, adiabatic ionization energy and vertical ionization energy. The former in the gas phase has been measured by photoelectron spectroscopy ${ }^{1,2}$ and the value in the solution phase has been also estimated from redox potential. ${ }^{3}$ On the other hand, vertical ionization energy had been measured only in the gas phase because of experimental difficulty in solution phase. Winter et al. recently measured the vertical ionization energy of various molecules (e.g., water, halogen anion, alkali cation, and transition metal complex) in aqueous solution. ${ }^{4-9}$ They show that the energy in aqueous solution is significantly different from the gas-phase value. The spectral width of vertical ionization is also reported, indicating that the width in the solution phase is significantly broadened caused by thermal fluctuation of solvent molecule.

The difference between vertical and adiabatic ionization in the gas phase is simply attributed to the molecular structure. Upon ionization, the structure remains unchanged (vertical ionization), and then relaxes toward the equilibrium structure in the ionized state (adiabatic ionization). On the other hand, because a molecule in solution phase strongly interacts with the surrounding solvent molecules, the ionization is deeply related to their thermal fluctuation, i.e., solvation structure. Marcus is the first to realize the importance of the fluctuation of solvent molecules upon electron transfer reaction, and proposed the concept of free energy surface. ${ }^{10,11}$ In this concept, the thermal fluctuation is expressed along the "solvation coordinate", on which the free energy of the system is projected. Note that because the ionization corresponds to the oxidative half reaction of electron transfer, the concept is also valid to treat the ionization process. While a simple dielectric continuum model was employed in the

\footnotetext{
a) Author to whom correspondence should be addressed. FAX: +81-(0)75383-2799. Electronic mail: hirofumi@moleng.kyoto-u.ac.jp.
}

original Marcus scheme, the same concept has been utilized in the molecular level computations such as molecular dynamics (MD) simulation, ${ }^{12-15}$ and the procedures ${ }^{16-19}$ based on the reference interaction site model (RISM) theory. ${ }^{20,21} \mathrm{~A}$ related quantity such as solvent reorganization energy $(\lambda)$ is also adequately computed. ${ }^{22}$ These numerous studies show that the free energy surface is often well described by the linear response regime in spite of the complexity caused by a huge number of solvent molecules. ${ }^{12-15,23-25,28}$ But a direct comparison with experimental knowledge is still very limited, presumably because of the lack of related experimental data.

In this paper, we report a theoretical study of ionization process in solution phase. Based on the Marcus-like free energy surface, the ionization and following relaxation processes are discussed in connection with the role of solvation through the comparison with experimental data. To achieve the purpose, an accurate evaluation of free energy upon the ionization is essential. In other words, an appropriate ensemble of solvent configurations that are consistent with the change in the electronic structure of solute molecule is required. RISM-SCF, ${ }^{26,27}$ a hybrid method of RISM theory ${ }^{20,21}$ and ab initio electronic structure theory, is regarded as an alternative to quantum mechanics/molecular mechanics. Using RISM-SCF, the electronic structure of solute molecule and solvation structure around it are obtained in a self-consistent manner. RISM theory is statistical mechanics for molecular liquid, and treats an ensemble of an infinite number of solvent molecules. It enables us to obtain the molecular level insight into the solvation effect. In addition, it is possible to combine with highly sophisticated electronic structure theory because the computational cost of the RISM theory is much lower than that of MD simulation. Recently, RISM-SCF is extended to the new version in which spacial electron density distribution (SEDD) is explicitly taken into account (RISM-SCF-SEDD method). ${ }^{29}$

The article is organized as follows. In Sec. II, a computational procedure for ionization energy in solution phase is explained. A new formula for the vertical ionization energy is developed with the aid of dielectric continuum theory ${ }^{30-32}$ in terms of the solvent relaxation process. ${ }^{73-37}$ 


\section{In the gas phase}

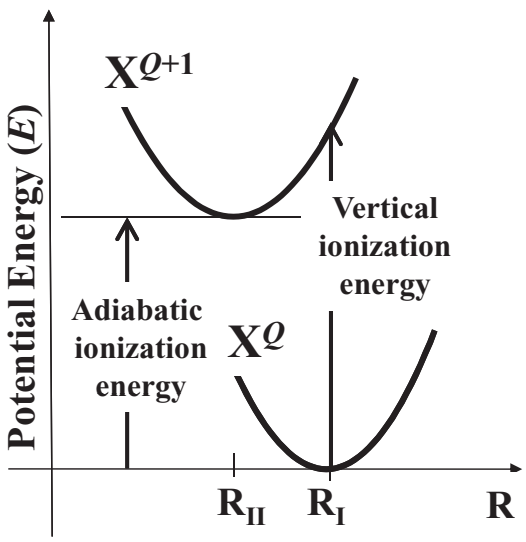

FIG. 1. Ionization process in the gas phase.

The computational results are presented in Secs. III and IV, followed by the conclusion in Sec. V.

\section{THEORETICAL METHOD}

\section{A. Ionization process in the gas phase}

Let us first review the ionization of a molecule $\mathrm{X}$ with total charge $Q\left(\mathrm{X}^{Q}\right)$ in the gas phase,

$$
\mathrm{X}^{Q} \rightarrow \mathrm{X}^{Q+1}+e^{-} .
$$

The ionization energy is related to the molecular geometry described by a set of all the atomic coordinates $(\{\mathbf{R}\})$. Figure 1 shows ionization process in the gas phase. The lower is the potential energy surface of $\mathrm{X}^{Q}$ and the upper one is that of the ionized species $\left(\mathrm{X}^{Q+1}\right)$ after the electron detachment. $\left\{\mathbf{R}_{\mathrm{I}}\right\}$ is the equilibrium geometry of $\mathrm{X}^{Q}$. The potential energy difference between $\mathrm{X}^{Q+1}$ and $\mathrm{X}^{Q}$ at the fixed geometry corresponds to the vertical ionization energy in the gas phase,

$$
I_{\mathrm{ver}}^{\mathrm{g}}=E^{+}\left(\left\{\mathbf{R}_{\mathrm{I}}\right\}\right)-E^{0}\left(\left\{\mathbf{R}_{\mathrm{I}}\right\}\right),
$$

where superscripts 0 and + , respectively, denote the state of $\mathrm{X}^{Q}$ and $\mathrm{X}^{Q+1}, E^{0}$ and $E^{+}$are their total energies. After the ionization, the molecule starts to relax and reaches the new equilibrium geometry $\left(\left\{\mathbf{R}_{\mathrm{II}}\right\}\right)$. The adiabatic ionization energy in the gas phase is defined by

$$
I_{\mathrm{ad}}^{\mathrm{g}}=E^{+}\left(\left\{\mathbf{R}_{\mathrm{II}}\right\}\right)-E^{0}\left(\left\{\mathbf{R}_{\mathrm{I}}\right\}\right) .
$$

\section{B. Ionization process in solution phase}

In solution phase, a set of all the coordinates of solvent molecule $(\{\mathbf{r}\})$ is also necessary to be considered. Instead of using this huge number of variables, it is convenient to introduce solvation coordinate $S .{ }^{12}$ Free energy surface in the vicinity of equilibrium state is expressed as a function of $S$,

$$
A^{x}(S)=A_{\min }^{x}-\frac{1}{\beta} \ln P^{x}(S),
$$

where superscript $x$ denotes the charge state 0 or,$+ \beta$ $=1 / k_{B} T$, and $k_{B}$ is the Boltzmann constant. $A_{\min }^{x}$ corresponds to the minimum of the free energy curvature. ${ }^{24} P^{x}(S)$ is the probability to find the system where $S$ equals the difference in the total energy of the system between the neutral and ionized states $(\Delta E \equiv \Delta E(\{\mathbf{R}\},\{\mathbf{r}\}))$,

$$
\begin{aligned}
P^{x}(S) & =\langle\delta[S-\Delta E(\{\mathbf{R}\},\{\mathbf{r}\})]\rangle_{x} \\
& =\frac{\int \delta[S-\Delta E(\{\mathbf{R}\},\{\mathbf{r}\})] \exp \left\{-\beta\left[E^{x}(\{\mathbf{R}\},\{\mathbf{r}\})\right]\right\} \mathrm{d}\{\mathbf{R}\} \mathrm{d}\{\mathbf{r}\}}{\int \exp \left\{-\beta\left[E^{x}(\{\mathbf{R}\},\{\mathbf{r}\})\right]\right\} \mathrm{d}\{\mathbf{R}\} \mathrm{d}\{\mathbf{r}\}},
\end{aligned}
$$

where $\delta$ is delta function, and $\langle\ldots\rangle_{x}$ denotes the ensemble average with state $x$. It is often approximated with Gaussian function, ${ }^{25}$

$$
P^{x}(S)=\frac{1}{\sqrt{2 \pi} \sigma_{x}} \exp \left\{-\frac{\left(S-\langle\Delta E\rangle_{x}\right)^{2}}{2 \sigma_{x}^{2}}\right\}
$$

The variance $\sigma_{x}$ is directly related to the spectral width of $I_{\text {ver }}{ }^{5,6}$ Figure 2 illustrates the ionization process in solution. Different from the gas phase phenomena shown in Figure 1, the horizontal and vertical axes, respectively, represent solvation coordinate $S$ and free energy. The surface of $\mathrm{X}^{Q}$ has the minimum at $S_{\mathrm{I}}$. Since the vertical ionization process occurs at the solvation of $S_{\text {I }}$ with the highest probability, the observed
$I_{\mathrm{ver}}$ is given by

$$
I_{\mathrm{ver}}=A^{+}\left(S_{\mathrm{I}}\right)-A^{0}\left(S_{\mathrm{I}}\right)=\langle\Delta E\rangle_{0} .
$$

After the vertical ionization, $S$ is changed through the relaxation of solvent configuration. The surface of $\mathrm{X}^{Q+1}$ has the minimum at $S_{\mathrm{II}}$. Adiabatic ionization energy is obtained as the free energy difference between the two minimum. ${ }^{23,24,38}$

$$
I_{\mathrm{ad}}=A^{+}\left(S_{\mathrm{II}}\right)-A^{0}\left(S_{\mathrm{I}}\right) .
$$

\section{Vertical ionization energy}

RISM-SCF-SEDD is an efficient method as far as equilibrium process is concerned and $I_{\mathrm{ad}}$ is directly evaluated from 


\section{In solution}

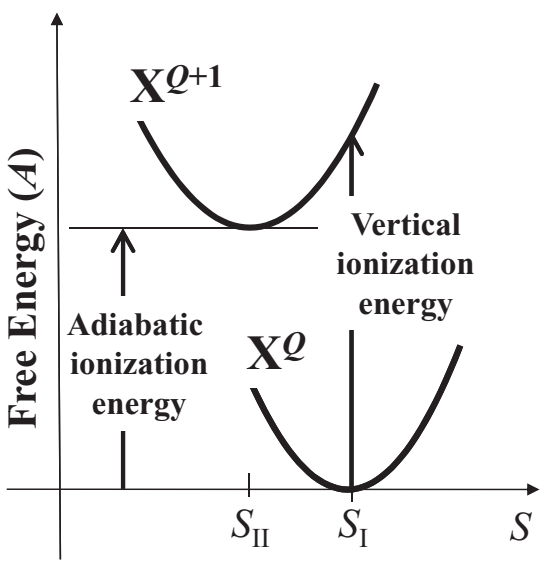

FIG. 2. Ionization process in solution.

Eq. (8). However, $I_{\mathrm{ver}}$ cannot be calculated with the original procedure because $\mathrm{X}^{Q+1}$ at $S_{\mathrm{I}}$ is not in equilibrium state. In the present study, the generalization is considered based on the charging formula with the aid of generalized Born theory. ${ }^{31,32}$ The analogous procedure is applied to polarizable continuum model (PCM) method based on the similarity in the treatment of electrostatic interaction between solute and solvent. $^{22,39}$

Let us first consider a set of vectors, $\mathbf{Q}^{0}$ and $\mathbf{Q}^{+}$, respectively, corresponding to neutral and ionized states. The vector component is the charge assigned to each atom (site) in the focused solute molecule. The solute-solvent electrostatic interaction potential generated on this site is also represented as a vector $(\mathbf{V})$. It is noted that the following discussion is applicable to any kind of theoretical framework, and an extension to treat continuous electronic distribution of the solute molecule is straightforward. To treat the vertical ionization, $\mathbf{V}$ is divided into two components in terms of the relaxation time,

$$
\mathbf{V}=\mathbf{V}_{\mathrm{f}}+\mathbf{V}_{\mathrm{s}} .
$$

The first term of the right hand side is fast component that immediately responds to the ionization. The second term corresponds to the slow component that remains unchanged just after the ionization. With Eq. (9), $I_{\mathrm{ver}}$ is given as the so-called charging formula with respect to a set of charges $(\mathbf{Q})$,

$$
\begin{aligned}
I_{\mathrm{ver}} & =A^{+}\left(S_{\mathrm{I}}\right)-A^{0}\left(S_{\mathrm{I}}\right) \\
& =\left\{U^{+}\left(S_{\mathrm{I}}\right)-U^{0}\left(S_{\mathrm{I}}\right)\right\}+\int_{\mathbf{Q}^{0}}^{\mathbf{Q}^{+}}\left\{\mathbf{V}_{\mathrm{f}}\left(S_{\mathrm{I}}\right)+\mathbf{V}_{\mathrm{s}}\left(S_{\mathrm{I}}\right)\right\} d \mathbf{Q},
\end{aligned}
$$

where $U^{+}$and $U^{0}$ are the internal energies of solute molecule, respectively. Because of the difference in the response of the field, $\mathbf{V}_{\mathrm{f}}$ and $\mathbf{V}_{\mathrm{s}}$, the integrations are separately performed. The slow component $\mathbf{V}_{\mathrm{s}}$ remains in the first state $\left(\mathbf{V}_{\mathrm{s}}^{0}\right)$ just after the vertical ionization, the integration over $\mathbf{V}_{\mathrm{s}}$ yields

$$
\int_{\mathbf{Q}^{0}}^{\mathbf{Q}^{+}} \mathbf{V}_{\mathrm{s}}\left(S_{\mathrm{I}}\right) d \mathbf{Q} \simeq\left\{\mathbf{Q}^{+}\left(S_{\mathrm{I}}\right)-\mathbf{Q}^{0}\left(S_{\mathrm{I}}\right)\right\} \cdot \mathbf{V}_{\mathrm{s}}^{0}\left(S_{\mathrm{I}}\right)
$$

$\mathbf{V}_{\mathrm{s}}^{0}$ is the slow part of the potential for the neutral state $\left(\mathbf{Q}^{0}\right)$. On the other hand, the fast component $\mathbf{V}_{\mathrm{f}}$ could change during the vertical ionization. If the linear dependency of $\mathbf{V}_{\mathrm{f}}$ on $\mathbf{Q}$ is assumed on the vertical ionization, then the integration of $\mathbf{V}_{f}$ yields

$$
\int_{\mathbf{Q}^{0}}^{\mathbf{Q}^{+}} \mathbf{V}_{\mathrm{f}}\left(S_{\mathrm{I}}\right) d \mathbf{Q} \simeq \frac{1}{2}\left\{\mathbf{Q}^{+}\left(S_{\mathrm{I}}\right) \cdot \mathbf{V}_{\mathrm{f}}^{+}\left(S_{\mathrm{I}}\right)-\mathbf{Q}^{0}\left(S_{\mathrm{I}}\right) \cdot \mathbf{V}_{\mathrm{f}}^{0}\left(S_{\mathrm{I}}\right)\right\} .
$$

From Eqs. (9)-(12), the following formula is obtained:

$$
\begin{aligned}
I_{\mathrm{ver}}= & \left\{U^{+}\left(S_{\mathrm{I}}\right)-U^{0}\left(S_{\mathrm{I}}\right)\right\}+\left\{\mathbf{Q}^{+}\left(S_{\mathrm{I}}\right)-\mathbf{Q}^{0}\left(S_{\mathrm{I}}\right)\right\} \cdot \mathbf{V}_{\mathrm{s}}^{0}\left(S_{\mathrm{I}}\right) \\
& +\frac{1}{2}\left\{\mathbf{Q}^{+}\left(S_{\mathrm{I}}\right) \cdot \mathbf{V}_{\mathrm{f}}^{+}\left(S_{\mathrm{I}}\right)-\mathbf{Q}^{0}\left(S_{\mathrm{I}}\right) \cdot \mathbf{V}_{\mathrm{f}}^{0}\left(S_{\mathrm{I}}\right)\right\} .
\end{aligned}
$$

With the aid of dielectric continuum theory, a further simple and practical equation is obtained based on Eq. (13). In the theory, the electrostatic potential ( $\mathbf{V}^{\text {dielec}}$; Refs. 34 and 40 ) is divided into fast and slow components as follows:

$$
\mathbf{V}_{\mathrm{f}}^{\text {dielec }}=k_{\mathrm{f}} \mathbf{V}^{\text {dielec }}, \quad \mathbf{V}_{\mathrm{s}}^{\text {dielec }}=k_{\mathrm{s}} \mathbf{V}^{\text {dielec }},
$$

where $k_{\mathrm{f}}$ and $k_{\mathrm{s}}$ are the constants given by

$$
k_{\mathrm{f}}=\left(1-\frac{1}{\epsilon_{\infty}}\right)\left(1-\frac{1}{\epsilon}\right)^{-1}, \quad k_{\mathrm{s}}=\left(\frac{1}{\epsilon_{\infty}}-\frac{1}{\epsilon}\right)\left(1-\frac{1}{\epsilon}\right)^{-1} .
$$

$\epsilon$ is dielectric permittivity and $\epsilon_{\infty}$ is optical dielectric permittivity. In dielectric continuum theory, the slow component is considered to be the orientational polarization of solvent as well as a part of electronic polarization of solvent. The fast component is related to the electronic polarization due to the change in the electrostatic field created by the solute. ${ }^{32,41}$ Since the electrostatic potential $\mathbf{V}$ is a common quantity among various theoretical frameworks, Eqs. (14) and (15) might be applicable to treat $\mathbf{V}$ in general,

$$
\mathbf{V}_{\mathrm{f}}=k_{\mathrm{f}} \mathbf{V}, \quad \mathbf{V}_{\mathrm{s}}=k_{\mathrm{s}} \mathbf{V} .
$$

Equation (13) is then rewritten as

$$
\begin{aligned}
I_{\text {ver }}= & \left\{U^{+}\left(S_{\mathrm{I}}\right)-U^{0}\left(S_{\mathrm{I}}\right)\right\}+k_{\mathrm{s}}\left\{\mathbf{Q}^{+}\left(S_{\mathrm{I}}\right)-\mathbf{Q}^{0}\left(S_{\mathrm{I}}\right)\right\} \cdot \mathbf{V}^{0}\left(S_{\mathrm{I}}\right) \\
& +\frac{1}{2} k_{\mathrm{f}}\left\{\mathbf{Q}^{+}\left(S_{\mathrm{I}}\right) \cdot \mathbf{V}^{+}\left(S_{\mathrm{I}}\right)-\mathbf{Q}^{0}\left(S_{\mathrm{I}}\right) \cdot \mathbf{V}^{0}\left(S_{\mathrm{I}}\right)\right\}
\end{aligned}
$$

In this equation, only $\mathbf{V}$ is necessary, and neither $\mathbf{V}_{\mathrm{f}}$ nor $\mathbf{V}_{\mathrm{s}}$ is required. It should be mentioned that $\mathbf{V}$ is, indeed, well evaluated with non-polarizable solvent model such as SPC model. ${ }^{42}$ This is because the model is considered to implicitly treat the electronic polarization of solvent through its empirical parameterization. ${ }^{42,43}$ Further detailed discussions are found in several literatures ${ }^{32,41}$ including recent report by Leontyev et al. ${ }^{44,45}$

According to Åqvist et al. ${ }^{25}$ the variance $\left(\sigma_{x}, x=0,+\right)$ is calculated based on Eq. (6) as follows:

$$
\beta \sigma_{x}^{2}=\left|\langle\Delta E\rangle_{0}-\langle\Delta E\rangle_{+}\right|
$$

The first term $\left(\langle\Delta E\rangle_{0}\right)$ is directly computed with Eqs. (7) and (17), and the second term is evaluated in a 
similar manner,

$$
\begin{aligned}
\langle-\Delta E\rangle_{+}= & A^{0}\left(S_{\mathrm{II}}\right)-A^{+}\left(S_{\mathrm{II}}\right) \\
= & \left\{U^{0}\left(S_{\mathrm{II}}\right)-U^{+}\left(S_{\mathrm{II}}\right)\right\} \\
& +k_{\mathrm{S}}\left\{\mathbf{Q}^{0}\left(S_{\mathrm{II}}\right)-\mathbf{Q}^{+}\left(S_{\mathrm{II}}\right)\right\} \cdot \mathbf{V}^{+}\left(S_{\mathrm{II}}\right) \\
& +\frac{1}{2} k_{\mathrm{f}}\left\{\mathbf{Q}^{0}\left(S_{\mathrm{II}}\right) \cdot \mathbf{V}^{0}\left(S_{\mathrm{II}}\right)-\mathbf{Q}^{+}\left(S_{\mathrm{II}}\right) \cdot \mathbf{V}^{+}\left(S_{\mathrm{II}}\right)\right\} .
\end{aligned}
$$

$\sigma_{x}$ can be then computed as the sum of these two terms. Therefore, the free energy surface within the linear response regime is fully elucidated from a set of information at the specific states.

\section{RISM-SCF-SEDD method}

In the present study, the original RISM-SCF-SEDD (Refs. 26, 27, and 29) was employed to calculate $I_{\mathrm{ad}}$ while the extended formula of RISM-SCF-SEDD was used to obtain $I_{\text {ver, }}$, as is noted in Sec. II C. RISM theory is given as follows: $:^{20,21}$

$$
\rho \mathbf{h} \rho=\omega * \mathbf{c} * \omega+\omega * \mathbf{c} * \rho \mathbf{h} \rho,
$$

where $*$ denotes convolution integral. The matrix element of $\rho$ is number density, $\omega$ represents intramolecular correlation function defining the molecular geometry, $\mathbf{c}$ is the direct correlation function, and $\mathbf{h}$ is the total correlation function, respectively. Because two unknown functions (i.e., $\mathbf{h}$ and $\mathbf{c}$ ) are included in Eq. (20), another equation relating these functions is required. A typical one is hyper-netted chain (HNC) closure,

$$
\begin{aligned}
c_{\alpha \lambda}(r)= & \exp \left[-\beta u_{\alpha \lambda}(r)+h_{\alpha \lambda}(r)-c_{\alpha \lambda}(r)\right] \\
& -\left\{h_{\alpha \lambda}(r)-c_{\alpha \lambda}(r)\right\}-1 .
\end{aligned}
$$

$\alpha$ and $\lambda$ are solute and solvent sites, respectively, and $u_{\alpha \lambda}(r)$ is interaction between the sites $\alpha$ and $\lambda$. Using Eqs. (20) and (21), an unique solvation structure is determined with respect to the given intermolecular interaction potential. The excess chemical potential (solvation free energy), $\Delta \mu$, is analytically calculated with the obtained $\mathbf{h}$ and $\mathbf{c},{ }^{46}$

$$
\Delta \mu=-\sum_{\alpha \lambda} \frac{\rho_{\lambda}}{\beta} \int \mathrm{d} \mathbf{r}\left[c_{\alpha \lambda}(r)-\frac{1}{2} h_{\alpha \lambda}^{2}(r)+\frac{1}{2} h_{\alpha \lambda}(r) c_{\alpha \lambda}(r)\right] .
$$

In RISM-SCF-SEDD, total free energy of the solution system is defined as

$$
A=E+\Delta \mu,
$$

where $E$ is the energy of solute molecule given as

$$
E=\langle\Phi|\hat{H}| \Phi\rangle .
$$

Here, $\hat{H}$ is the standard electronic Hamiltonian of the solute molecule in the isolated state. $\Phi$ is the electronic wave function in solution phase, and is obtained based on the variational condition with respect to $A,{ }^{26,27,29}$

$$
\left\langle\delta \Phi\left|\hat{F}-\hat{V}^{\mathrm{R}}\right| \Phi\right\rangle=0,
$$

where $\hat{F}$ is Fock operator and $\hat{V}^{\mathrm{R}}$ is the solute-solvent electrostatic interaction operator in the framework of RISM-SCFSEDD. Note that $E$ in solution is generally different from that in the gas phase because the electrostatic field created by solvent induces the electronic polarization of solute molecule. The mean electrostatic interaction energy between solute and solvent is written as follows: ${ }^{29}$

$$
\left\langle\Phi\left|\hat{V}^{\mathrm{R}}\right| \Phi\right\rangle=\sum_{i} D_{i} V_{i}^{\mathrm{R}}=\mathbf{D} \cdot \mathbf{V}^{\mathrm{R}} .
$$

Here, $D_{i}$ is expansion coefficients of charge density $\rho(\mathbf{r})$ defined as

$$
\rho(\mathbf{r})=\sum_{i}^{N_{\mathrm{ABS}}} D_{i} f_{i}(\mathbf{r}),
$$

where $f_{i}(\mathbf{r})$ is auxiliary basis sets (ABSs) centered on each solute site and $N_{\mathrm{ABS}}$ is the number of ABSs. $\mathbf{D}$ is uniquely determined by the wave function $\Phi$. Here, the Gaussian function is employed as $f_{i}(\mathbf{r})$ to describe the electron distribution, and the delta function is employed to describe nucleus charge distribution. The element $D_{i}$ would be thus regarded as the net population of electron or nucleus charge belonging to $i$-th auxiliary basis. The effective electrostatic interaction potential between $f_{i}(\mathbf{r})$ and solvent is given by

$$
V_{i}^{\mathrm{R}}=\sum_{\lambda} \rho_{\lambda} \int \frac{f_{i}\left(\mathbf{r}^{\prime}-\mathbf{r}_{\alpha}\right)}{\left|\mathbf{r}^{\prime}-\mathbf{r}\right|} h_{\alpha \lambda}\left(\left|\mathbf{r}^{\prime}-\mathbf{r}\right|\right) d \mathbf{r} d \mathbf{r}^{\prime}, \quad(i \in \alpha) .
$$

In standard RISM-SCF-SEDD procedure, $\Phi$ and $\mathbf{V}^{\mathrm{R}}$ at the equilibrium state are determined in a self-consistent manner by iteratively solving RISM part (Eqs. (20) and (21)) and electronic structure calculation part (Eq. (25)). It should be stressed that $\mathbf{V}$ is computed based on Eq. (28) and molecular level information of solvation $\left(h_{\alpha \lambda}(r)\right)$ is necessary for the evaluation.

The adiabatic ionization energy in solution phase $\left(I_{\mathrm{ad}}\right)$ can be calculated with the two separated standard RISM-SCFSEDD calculations, i.e., for the neutral and cation species in the equilibrium state. On the other hand, it is generally difficult to compute $I_{\mathrm{ver}}$ with the standard procedure, because $\mathrm{X}^{Q+1}$ at $S_{\mathrm{I}}$ does not correspond to equilibrium state, and the electronic polarizability of solvent has non-negligible contribution. However, the analogous procedure based on Eq. (17) is possible to apply in RISM-SCF-SEDD method. In the equation, $\mathbf{V}$ and $\mathbf{Q}$ are, respectively, replaced with $\mathbf{V}^{\mathrm{R}}$ and $\mathbf{D}$. The internal energies, $U^{+}$and $U^{0}$, are replaced with the total energies of the solute molecule, $E^{+}$and $E^{0}$,

$$
\begin{aligned}
I_{\mathrm{ver}}= & \left\{E^{+}\left(S_{\mathrm{I}}\right)-E^{0}\left(S_{\mathrm{I}}\right)\right\}+k_{\mathrm{s}}\left\{\mathbf{D}^{+}\left(S_{\mathrm{I}}\right)-\mathbf{D}^{0}\left(S_{\mathrm{I}}\right)\right\} \cdot \mathbf{V}^{\mathrm{R}, 0}\left(S_{\mathrm{I}}\right) \\
& +\frac{1}{2} k_{\mathrm{f}}\left\{\mathbf{D}^{+}\left(S_{\mathrm{I}}\right) \cdot \mathbf{V}^{\mathrm{R},+}\left(S_{\mathrm{I}}\right)-\mathbf{D}^{0}\left(S_{\mathrm{I}}\right) \cdot \mathbf{V}^{\mathrm{R}, 0}\left(S_{\mathrm{I}}\right)\right\} .
\end{aligned}
$$

Because the electronic structure of the solute molecule for $\mathrm{X}^{Q+1}$ depends on the generated electrostatic field, the variational condition is given as follows:

$$
\left\langle\delta \Phi\left|\hat{F}-\left(k_{\mathrm{f}} \hat{V}^{\mathrm{R},+}\left(S_{\mathrm{I}}\right)+k_{\mathrm{s}} \hat{V}^{\mathrm{R}, 0}\left(S_{\mathrm{I}}\right)\right)\right| \Phi\right\rangle=0 .
$$

$\mathbf{V}^{\mathrm{R},+}\left(S_{\mathrm{I}}\right)$ and $\mathbf{D}^{+}\left(S_{\mathrm{I}}\right)$ are computed self-consistently by iteratively solving the RISM part (Eqs. (20) and (21)) and the 
electronic structure part (Eq. (30)). $I_{\text {ver }}$ is thus computed with $E^{0}, \mathbf{D}^{0}\left(S_{\mathrm{I}}\right)$, and $\mathbf{V}^{\mathrm{R}, 0}\left(S_{\mathrm{I}}\right)$ obtained from the standard RISMSCF-SEDD procedure. It is therefore determined only with two additional parameters, $\epsilon$ and $\epsilon_{\infty}$, using the analogous procedure to the standard RISM-SCF-SEDD method. The spectral density $\left(\sigma_{x}\right)$ is similarly expressed as follows:

$$
\begin{aligned}
\beta \sigma_{x}^{2}= & \mid\left\{E^{+}\left(S_{\mathrm{I}}\right)-E^{0}\left(S_{\mathrm{I}}\right)\right\}+k_{\mathrm{S}}\left\{\mathbf{D}^{+}\left(S_{\mathrm{I}}\right)-\mathbf{D}^{0}\left(S_{\mathrm{I}}\right)\right\} \cdot \mathbf{V}^{\mathrm{R}, 0}\left(S_{\mathrm{I}}\right) \\
& +\frac{1}{2} k_{\mathrm{f}}\left\{\mathbf{D}^{+}\left(S_{\mathrm{I}}\right) \cdot \mathbf{V}^{\mathrm{R},+}\left(S_{\mathrm{I}}\right)-\mathbf{D}^{0}\left(S_{\mathrm{I}}\right) \cdot \mathbf{V}^{\mathrm{R}, 0}\left(S_{\mathrm{I}}\right)\right\} \\
& +\left\{E^{0}\left(S_{\mathrm{II}}\right)-E^{+}\left(S_{\mathrm{II}}\right)\right\} \\
& +k_{\mathrm{S}}\left\{\mathbf{D}^{0}\left(S_{\mathrm{II}}\right)-\mathbf{D}^{+}\left(S_{\mathrm{II}}\right)\right\} \cdot \mathbf{V}^{\mathrm{R},+}\left(S_{\mathrm{II}}\right) \\
& +\frac{1}{2} k_{\mathrm{f}}\left\{\mathbf{D}^{0}\left(S_{\mathrm{II}}\right) \cdot \mathbf{V}^{\mathrm{R}, 0}\left(S_{\mathrm{II}}\right)-\mathbf{D}^{+}\left(S_{\mathrm{II}}\right) \cdot \mathbf{V}^{\mathrm{R},+}\left(S_{\mathrm{II}}\right)\right\} \mid .
\end{aligned}
$$

A further simplified expression without dividing into the fast and slow components is available by setting $\epsilon_{\infty}=1$ in Eq. (29),

$$
I_{\text {ver }}^{\mathrm{wd}}=\left\{E^{+}\left(S_{\mathrm{I}}\right)-E^{0}\left(S_{\mathrm{I}}\right)\right\}+\left\{\mathbf{D}^{+}\left(S_{\mathrm{I}}\right)-\mathbf{D}^{0}\left(S_{\mathrm{I}}\right)\right\} \cdot \mathbf{V}^{\mathrm{R}, 0}\left(S_{\mathrm{I}}\right) .
$$

This formula is essentially the same as previously used one to deal with the vertical excitation in solution phase. ${ }^{16-19}$

\section{COMPUTATIONAL DETAIL}

In this study, we examined the ionization of cations,

$\mathrm{Li}^{+} \rightarrow \mathrm{Li}^{2+}+\mathrm{e}^{-}, \quad \mathrm{Na}^{+} \rightarrow \mathrm{Na}^{2+}+\mathrm{e}^{-}, \mathrm{K}^{+} \rightarrow \mathrm{K}^{2+}+\mathrm{e}^{-}$, anions,

$$
\begin{aligned}
& \mathrm{F}^{-} \rightarrow \mathrm{F}+\mathrm{e}^{-}, \quad \mathrm{Cl}^{-} \rightarrow \mathrm{Cl}+\mathrm{e}^{-}, \quad \mathrm{Br}^{-} \rightarrow \mathrm{Br}+\mathrm{e}^{-}, \\
& \mathrm{I}^{-} \rightarrow \mathrm{I}+\mathrm{e}^{-},
\end{aligned}
$$

and water molecule,

$$
\mathrm{H}_{2} \mathrm{O} \rightarrow \mathrm{H}_{2} \mathrm{O}^{+}+\mathrm{e}^{-} \text {. }
$$

Geometry optimization of $\mathrm{H}_{2} \mathrm{O}$ was performed by $\mathrm{B} 3 \mathrm{LYP} / 6-311++\mathrm{G}^{* *}$. Energy calculations were then carried out at MP2/6-311++G** level. For ionized open shell species, the unrestricted treatment (UB3LYP, UHF, and UMP2) were adopted. Solvation effect was taken into account using RISM-SCFSEDD method and PCM. ${ }^{32}$ Non-equilibrium PCM (Ref. 34) was employed for the calculations of $I_{\mathrm{ver}}$. Gas phase and PCM calculations were performed by GAUSSIAN 03 program package ${ }^{47}$ while RISM-SCF-SEDD calculations were done by GAMESS package ${ }^{48}$ modified by us.

In the RISM procedure, the density of solvent water was set to $1.00 \mathrm{~g} / \mathrm{cm}^{3}$ at $T=298.15 \mathrm{~K}$, and HNC closure ${ }^{46}$ was employed. The Lennard-Jones (L-J) parameters of the solutes were taken from Refs. 49 and 50 and SPC-like water was employed for the solvent. ${ }^{42}$ All of them are summarized in Table I. Note that the non-electrostatic interaction described by the L-J potential is much smaller than the electronic interaction between ion and water. The dependency on the L-J parameter is considered to be small enough. Dielectric per-
TABLE I. Lennard-Jones parameters.

\begin{tabular}{lcc}
\hline \hline & $\sigma(\AA)$ & $\epsilon\left(\mathrm{kcal} \mathrm{mol}^{-1}\right)$ \\
\hline $\mathrm{Li}$ & 1.394 & 0.128 \\
$\mathrm{Na}$ & 2.274 & 0.116 \\
$\mathrm{~K}$ & 3.154 & 0.111 \\
$\mathrm{~F}$ & 2.720 & 0.348 \\
$\mathrm{Cl}$ & 3.620 & 0.448 \\
$\mathrm{Br}$ & 3.900 & 0.658 \\
$\mathrm{I}$ & 4.320 & 0.806 \\
$\mathrm{O}$ & 3.166 & 0.155 \\
$\mathrm{H}$ & 1.000 & 0.056 \\
$\mathrm{O}^{\mathrm{a}}$ & 3.166 & 0.155 \\
$\mathrm{H}^{\mathrm{a}}$ & 1.000 & 0.056 \\
\hline \hline
\end{tabular}

mittivity and optical dielectric permittivity of water solvent were set to $\epsilon=78.390$ and $\epsilon_{\infty}=1.776 .{ }^{47}$

\section{RESULTS AND DISCUSSIONS}

\section{A. Ionization energy}

Table II shows ionization energy in the gas phase $\left(I_{\mathrm{ver}}^{\mathrm{g}}\right)$ and the difference from the experimental value. ${ }^{51,52}$ Although the computed values are slightly greater than the experimental ones, the root-mean-square of the deviations is $0.3 \mathrm{eV}$, showing a reasonable agreement. Table III shows the change of ionization energy caused by solvation, $\Delta I_{\mathrm{ver}}\left(=I_{\mathrm{ver}}-I_{\mathrm{ver}}^{\mathrm{g}}\right)$ and $\Delta I_{\mathrm{ad}}\left(=I_{\mathrm{ad}}-I_{\mathrm{ad}}^{\mathrm{g}}\right)$, which are the central results in this study. The corresponding experimental values ${ }^{3-5}$ and $\Delta I_{\mathrm{ver}}^{\mathrm{wd}}$ (Eq. (32)) are also listed. It is noted that the contributions from the solvation $\left(\Delta I_{\mathrm{ver}}\right.$ and $\left.\Delta I_{\mathrm{ad}}\right)$ are much greater than the deviations found in Table II. Clearly, the computational results on the solvation show good agreement with experimental values and well reproduce the dependencies on the solute size: As large the size of molecule, the absolute value of $\Delta I_{\mathrm{ver}}$ and $\Delta I_{\mathrm{ad}}$ decreases. It is negative for cation whereas positive for anion.

By definition, the values of $\Delta I_{\text {ad }}$ are a few eV lower than those of $\Delta I_{\mathrm{ver}}$, because of the stabilization caused by the relaxation of solvent configuration. This is well illustrated in

TABLE II. Vertical ionization energy in the gas phase $\left(I_{\text {ver }}^{\mathrm{g}}\right)$ by MP2 and experimental methods. ${ }^{51,52}$ The difference from the experimental value is also listed together with the root-mean-square deviation (RMSD). The unit is $\mathrm{eV}$.

\begin{tabular}{lccc}
\hline \hline & $\begin{array}{c}\text { Exp. } \\
\text { (Refs. 51 and 52) }\end{array}$ & MP2 & Deviation \\
\hline $\mathrm{Li}^{+} \rightarrow \mathrm{Li}^{2+}$ & 75.6 & 75.0 & 0.6 \\
$\mathrm{Na}^{+} \rightarrow \mathrm{Na}^{2+}$ & 47.3 & 47.0 & 0.3 \\
$\mathrm{~K}^{+} \rightarrow \mathrm{K}^{2+}$ & 31.6 & 31.3 & 0.3 \\
$\mathrm{~F}^{-} \rightarrow \mathrm{F}$ & 3.4 & 3.2 & 0.2 \\
$\mathrm{Cl}^{-} \rightarrow \mathrm{Cl}$ & 3.6 & 3.2 & 0.4 \\
$\mathrm{Br}^{-} \rightarrow \mathrm{Br}$ & 3.4 & 3.1 & 0.3 \\
$\mathrm{I}^{-} \rightarrow \mathrm{I}$ & 3.1 & 3.0 & 0.1 \\
$\mathrm{H}_{2} \mathrm{O} \rightarrow \mathrm{H}_{2} \mathrm{O}^{+}$ & 12.6 & 12.6 & 0.0 \\
$\mathrm{RMSD}$ (calculated from exp.) & & 0.3 \\
\hline
\end{tabular}


TABLE III. The change of ionization energy by solvation in $\mathrm{eV}$.

\begin{tabular}{|c|c|c|c|c|c|c|c|c|}
\hline & \multicolumn{2}{|c|}{ Experiment } & \multicolumn{3}{|c|}{ RISM - SCF - SEDD } & \multicolumn{3}{|c|}{ PCM } \\
\hline & $\Delta \mathrm{I}_{\mathrm{ver}}($ Refs. 4 and 5) & $\Delta \mathrm{I}_{\mathrm{ad}}($ Ref. 3) & $\Delta \mathrm{I}_{\mathrm{ver}}^{\mathrm{Wd}}$ & $\Delta \mathrm{I}_{\mathrm{ver}}$ & $\Delta \mathrm{I}_{\mathrm{ad}}$ & $\Delta \mathrm{I}_{\mathrm{ver}}^{\mathrm{wd}}$ & $\Delta \mathrm{I}_{\mathrm{ver}}$ & $\Delta \mathrm{I}_{\mathrm{ad}}$ \\
\hline $\mathrm{Li}^{+} \rightarrow \mathrm{Li}^{2+}$ & -15.2 & & -10.5 & -13.5 & -16.4 & -11.5 & -14.2 & -17.4 \\
\hline $\mathrm{Na}^{+} \rightarrow \mathrm{Na}^{2+}$ & -11.9 & & -8.0 & -10.3 & -12.6 & -9.4 & -11.6 & -14.3 \\
\hline $\mathrm{K}^{+} \rightarrow \mathrm{K}^{2+}$ & -9.4 & & -6.5 & -8.3 & -10.1 & -7.4 & -9.1 & -11.2 \\
\hline $\mathrm{F}^{-} \rightarrow \mathrm{F}$ & $(6.4)^{\mathrm{a}}$ & 4.8 & 11.2 & 8.6 & 5.4 & 8.0 & 6.2 & 4.0 \\
\hline $\mathrm{Cl}^{-} \rightarrow \mathrm{Cl}$ & 6.0 & 3.5 & 7.6 & 5.8 & 3.8 & 6.7 & 5.2 & 3.1 \\
\hline $\mathrm{Br}^{-} \rightarrow \mathrm{Br}$ & 5.4 & 3.2 & 7.0 & 5.4 & 3.5 & 6.1 & 4.7 & 2.9 \\
\hline $\mathrm{I}^{-} \rightarrow \mathrm{I}$ & 4.6 & 2.8 & 6.2 & 4.8 & 3.2 & 5.5 & 4.2 & 2.6 \\
\hline $\mathrm{H}_{2} \mathrm{O} \rightarrow \mathrm{H}_{2} \mathrm{O}^{+}$ & -1.5 & & 0.8 & -1.0 & -3.0 & -0.3 & -1.9 & -3.9 \\
\hline RMSD (w.r.t. exp.) & & & $\ldots$ & 1.2 & 0.4 & $\ldots$ & 0.6 & 0.5 \\
\hline
\end{tabular}

${ }^{\mathrm{a}}$ The experimental value of $\Delta I_{\mathrm{ver}}$ of $\mathrm{F}^{-}$is the estimated value., ${ }^{5,53}$

the pair correlation functions (PCFs) shown in Fig. 3. The first peak between $\mathrm{Na}^{+}$and water oxygen $\left(\mathrm{O}_{\mathrm{W}}\right)$ at $r=2 \AA$ gets higher after the ionization due to increase of the charge $\left(\mathrm{Na}^{+} \rightarrow \mathrm{Na}^{2+}\right)$. On the other hand, the first peak between $\mathrm{Cl}^{-}$and water hydrogen $\left(\mathrm{H}_{\mathrm{W}}\right)$ at $r=2 \AA$ corresponding

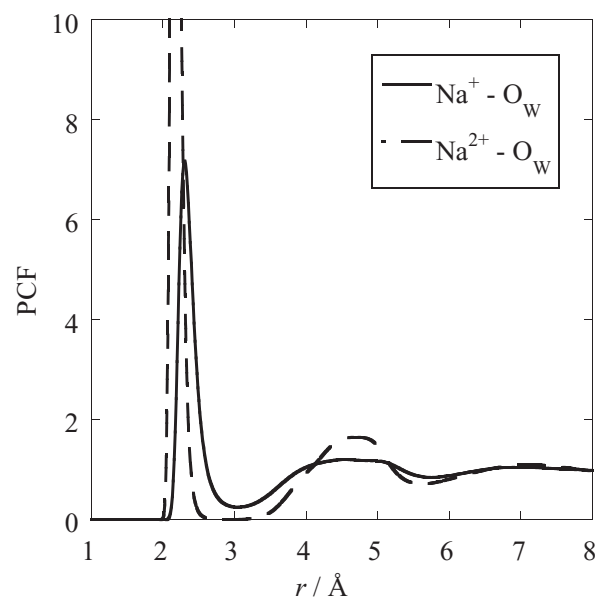

(a)

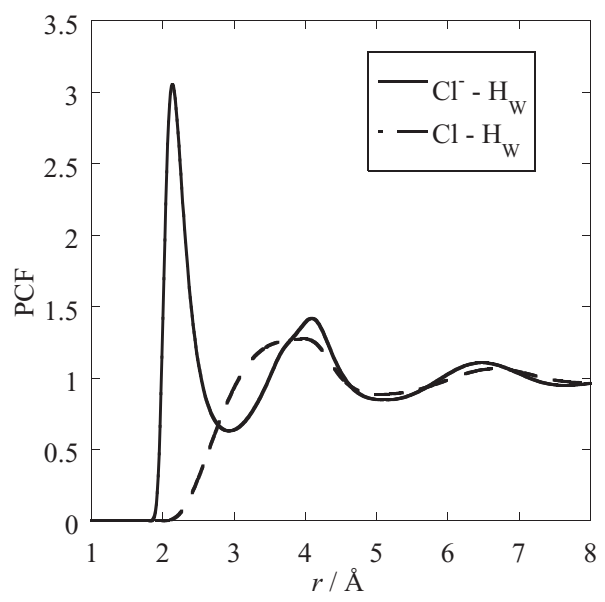

(b)

FIG. 3. (a) $\mathrm{PCF}$ of $\mathrm{Na}^{+}-\mathrm{O}_{\mathrm{W}}$, and that of $\mathrm{Na}^{2+}$ and $\mathrm{O}_{\mathrm{W}}$. (b) $\mathrm{PCF}$ of $\mathrm{Cl}^{-}-$ $\mathrm{H}_{\mathrm{W}}$, and that of $\mathrm{Cl}-\mathrm{H}_{\mathrm{W}}$. The solid lines are before ionization while dashed lines indicate after ionization. to $\mathrm{Cl}^{-}-\mathrm{H}_{\mathrm{W}}$ hydrogen bond completely disappears after the ionization, i.e., the detachment of electron from $\mathrm{Cl}^{-}\left(\mathrm{Cl}^{-}\right.$ $\rightarrow \mathrm{Cl}$ ). These remarkable changes of solvation structure are consistent with the dominant stabilization by orientational polarization.

\section{B. Solute-solvent electrostatic interaction}

The first two terms in Eqs. (29) and (32), corresponding to the polarization of solvent, comprise only less than $5 \%$ of total $\Delta I_{\mathrm{ver}}$. For example, the first two terms of $\mathrm{Cl}^{-}$is $0.02 \mathrm{eV}$, which is much smaller than the total energy $(5.8 \mathrm{eV})$. In other words, $\Delta I_{\text {ver }}$ is mainly determined by the remaining component that originates from the changing in the solute-solvent electrostatic interaction energy.

In general, electrostatic interaction energy is expanded by multipole moments. For a charged molecule $(Q \neq 0)$, the monopole term $(Q V)$ is dominative. Based on Eq. (29), $\Delta I_{\text {ver }}$ is approximated as

$$
\Delta I_{\mathrm{ver}} \simeq k_{\mathrm{s}}\left\{Q^{+}-Q^{0}\right\} V^{0}\left(S_{\mathrm{I}}\right)+\frac{1}{2} k_{\mathrm{f}}\left\{Q^{+} V^{+}\left(S_{\mathrm{I}}\right)-Q^{0} V^{0}\left(S_{\mathrm{I}}\right)\right\},
$$

where $Q^{x}(x=0$ or +$)$ is the solute charge in the state $x$, and $V_{x}$ is the electrostatic field generated by the solvent with this state. According to the dielectric continuum theory, the reaction field $V^{x}$ is given as follows: ${ }^{30}$

$$
V^{x}=-\left(1-\frac{1}{\epsilon}\right) \frac{Q^{x}}{a},
$$

where $a$ is solute radius. Using this equation, Eq. (33) is rewritten as an explicit function of $Q^{0}$ and $Q^{+} . \Delta I_{\mathrm{ver}}^{\mathrm{wd}}$ is also in accord with the experimental value and rewritten as

$$
\Delta I_{\mathrm{ver}}^{\mathrm{wd}}=\left(Q^{+}-Q^{0}\right) V^{0}\left(S_{\mathrm{I}}\right)=-\left(Q^{+}-Q^{0}\right)\left(1-\frac{1}{\epsilon}\right) \frac{Q^{0}}{a} .
$$

This simple equation may be useful to understand the computational results shown in Table III, which are obtained from highly sophisticated theory. Equation (35) of cation $\left(Q^{0}=1\right.$ and $Q^{+}=2$ ) becomes negative whereas that of anion $\left(Q^{0}=-1\right.$ and $\left.Q^{+}=0\right)$ is positive, and the absolute value 
TABLE IV. Full width at half maximum of vertical ionization energy in aqueous solution. The unit is eV.

\begin{tabular}{lcccccc}
\hline \hline & & \multicolumn{2}{c}{ RISM-SCF - SEDD } & & \multicolumn{2}{c}{ PCM } \\
\cline { 3 - 4 } \cline { 6 - 7 } & Experiment ${ }^{4,5}$ & $\epsilon_{\infty}=1.000$ & $\epsilon_{\infty}=1.776$ & & $\epsilon_{\infty}=1.000$ & $\epsilon_{\infty}=1.776$ \\
\hline $\mathrm{Li}^{+} \rightarrow \mathrm{Li}^{2+}$ & $1.4 \pm 0.2$ & 1.3 & 1.0 & & 1.3 & 1.0 \\
$\mathrm{Na}^{+} \rightarrow \mathrm{Na}^{2+}$ & $1.1 \pm 0.3$ & 1.1 & 0.9 & & 1.2 & 0.9 \\
$\mathrm{~K}^{+} \rightarrow \mathrm{K}^{2+}$ & $1.4 \pm 0.2$ & 1.0 & 0.8 & & 1.0 & 0.8 \\
$\mathrm{~F}^{-} \rightarrow \mathrm{F}$ & & 1.2 & 0.9 & & 1.1 & 0.8 \\
$\mathrm{Cl}^{-} \rightarrow \mathrm{Cl}$ & $0.6 \pm 0.2$ & 1.0 & 0.8 & & 1.0 & 0.8 \\
$\mathrm{Br}^{-} \rightarrow \mathrm{Br}$ & $0.9 \pm 0.2$ & 1.0 & 0.7 & & 0.9 & 0.7 \\
$\mathrm{I}^{-} \rightarrow \mathrm{I}$ & $0.8 \pm 0.3$ & 0.9 & 0.7 & & 0.9 & 0.7 \\
$\mathrm{H}_{2} \mathrm{O} \rightarrow \mathrm{H}_{2} \mathrm{O}^{+}$ & 1.45 & 1.0 & 0.8 & & 1.0 & 0.8 \\
$\mathrm{RMSD}$ (calculated from exp.) & 0.3 & 0.4 & & 0.3 & 0.4 \\
\hline \hline
\end{tabular}

of $\Delta I_{\mathrm{ver}}$ decreases as increase of $a$. Because of the neutrality, Eq. (34) for $\mathrm{H}_{2} \mathrm{O}\left(Q^{0}=0\right)$ becomes zero, and actually the absolute value of $\Delta I_{\mathrm{ver}}^{\mathrm{wd}}$ is much smaller than those of the ions. The difference between $\Delta I_{\mathrm{ver}}$ and $\Delta I_{\mathrm{ver}}^{\mathrm{wd}}$ allows us to estimate the polarization energy,

$$
\Delta I_{\mathrm{ver}}^{\mathrm{wd}}-\Delta I_{\mathrm{ver}}=\left(1-\frac{1}{\epsilon_{\infty}}\right) \frac{\left(Q^{+}-Q^{0}\right)^{2}}{2 a} .
$$

The quantity is always positive by definition.

\section{Spectral width of vertical ionization energy}

Table IV shows the full width at half maximum of $I_{\text {ver }}\left(2 \sqrt{2 \ln 2} \sigma_{0} \simeq 2.35 \sigma_{0}\right)$. The computed values are mostly within the limit of accuracy of the experimental values. The accordance is consistent with the result of MD simulation by Åqvist et al. ${ }^{25}$ showing that the free energy surfaces of the molecules investigated in the present study are well described by the linear response regime. Also, the free energy curvatures computed by the present method are similar to those by simulations. Based on Eqs. (33) and (34), $\sigma_{0}$ is approximated as

$$
\sigma_{0}=\sqrt{\frac{1}{\beta}\left(\frac{1}{\epsilon_{\infty}}-\frac{1}{\epsilon}\right) \frac{\left(Q^{+}-Q^{0}\right)^{2}}{a}}
$$

This equation indicates the width increases as the decrease of the solute size $(a)$. Although the difference originated from the size dependency is here in the range of experimental error bar, the size dependency given by Eq. (37) is consistent with the theoretical results.

Equation (37) is also rewritten as follows:

$$
\sigma_{0}=\sqrt{k_{\mathrm{s}}} \sqrt{\frac{1}{\beta}\left(1-\frac{1}{\epsilon}\right) \frac{\left(Q^{+}-Q^{0}\right)^{2}}{a}} .
$$

If the fast and the slow component are not divided, $\sqrt{k_{\mathrm{s}}}$ equals 1 and the width is represented only with the second squareroot part. If $\epsilon_{\infty}=1.776$ is assumed, $\sqrt{k_{\mathrm{s}}}$ equals 0.747 . This suggests that the contribution from fast component makes the spectra width narrower.

\section{CONCLUSION}

Ionization process in aqueous solution was studied using hybrid-type electronic structure theories, RISM-SCF-SEDD method and PCM. The vertical and adiabatic ionization were evaluated based on the free energy surface, and thereon the role of solvation was discussed. The computed values show well agreement with experimental measurements.

With the aid of dielectric continuum theory, the ionization energy was further analyzed. A semi-empirical formula, which allows us to divide the solute-solvent interaction into fast and slow components, was proposed. The solvation before ionization mainly determines the change of vertical ionization energy. However, the fast polarization of solvent induced by the ionization also has non-negligible contribution. In particular, the change of ionization energy of $\mathrm{H}_{2} \mathrm{O}$ is mainly determined by the fast polarization. On the other hand, the relaxation of solvent configuration, namely, the change of solvation structure, is the main origin of the change of the adiabatic ionization energy. The spectral width of the vertical ionization was also studied. The calculated widths were well in accord with the experimental values.

\section{ACKNOWLEDGMENTS}

We are grateful to Professor Benedetta Mennucci (Pisa University) for invaluable comments. K.I. thanks the grantin-aid for JSPS fellows. The work is financially supported in part by grant-in-aid for scientific research on priority areas "Molecular Science for Supra Functional Systems" (Grant No. 477-22018016), grant-in-aid for scientific research on innovative areas "Molecular Science of Fluctuations" (Grant No. 2006-21107511), as well as by grant-in-aid for scientific research (C) (Grant No. 20550013). The Strategic Programs for Innovative Research (SPIRE), the Computational Materials Science Initiative (CMSI), and the Ministry of Education, Culture, Sports, Science and Technology (MEXT) Japan are also acknowledged. Theoretical computations were partly performed using Research Center for Computational Science, Okazaki, Japan.

\footnotetext{
${ }^{1}$ K. M. Ervin, Chem. Rev. 101, 391 (2001).

${ }^{2}$ K. M. Dethlefs and E. W. Schlag, Annu. Rev. Phys. Chem. 42, 109 (1991).

${ }^{3}$ R. G. Pearson, J. Am. Chem. Soc. 108, 6109 (1986).
} 
${ }^{4}$ B. Winter, R. Weber, W. Widdra, M. Dittmar, M. Faubel, and I. V. Hertel, J. Phys. Chem. A 108, 2625 (2004).

${ }^{5}$ B. Winter, R. Weber, I. V. Hertel, M. Faubel, P. Jungwirth, E. C. Brown, and S. E. Bradforth, J. Am. Chem. Soc. 127, 7203 (2005).

${ }^{6}$ B. Winter and M. Faubel, Chem. Rev. 106, 1176 (2006).

${ }^{7}$ B. J. Cwiklik, P. Slavíček, L. Cwiklik, D. Nolting, B. Winter, and P. Jungwirth, J. Phys. Chem. A 112, 3499 (2008).

${ }^{8}$ R. Seidel, M. Faubel, B. Winter, and J. Blumberger, J. Am. Chem. Soc. 131, 16127 (2009).

${ }^{9}$ J. Moens, R. Seidel, P. Geerlings, M. Faubel, B. Winter, and J. Blumberger, J. Phys. Chem. B 114, 9173 (2010).

${ }^{10}$ R. A. Murcus, J. Chem. Phys. 24, 966 (1956).

${ }^{11}$ R. A. Murcus, J. Chem. Phys. 24, 979 (1956).

${ }^{12}$ A. Warshel and W. W. Parson, Annu. Rev. Phys. Chem. 42, 279 (1991).

${ }^{13}$ K. Ando and S. Kato, J. Chem. Phys. 95, 5966 (1991).

${ }^{14}$ G. King and A. Warshel, J. Chem. Phys. 93, 8682 (1990).

${ }^{15}$ E. A. Carter and J. T. Hynes, J. Phys. Chem. 93, 2184 (1989).

${ }^{16}$ H. Sato, Y. Kobori, S. Tero-Kubota, and F. Hirata, J. Chem. Phys. 119, 2753 (2003).

${ }^{17}$ H. Sato, Y. Kobori, S. Tero-Kubota, and F. Hirata, J. Phys. Chem. B 108, 11709 (2004).

${ }^{18}$ S. Chong, S. Miura, G. Basu, and F. Hirata, J. Phys. Chem. 99, 10526 (1995).

${ }^{19}$ N. Yoshida, T. Ishida, and F. Hirata, J. Phys. Chem. B 112, 433 (2008).

${ }^{20}$ D. Chandler and H. C. Andersen, J. Chem. Phys. 57, 1930 (1972).

${ }^{21}$ F. Hirata and P. J. Rossky, Chem. Phys. Lett. 83, 329 (1981).

${ }^{22}$ M. Caricato, F. Ingrosso, B. Mennucci, and H. Sato, J. Phys. Chem. B 110, 25115 (2006).

${ }^{23}$ J. Blumberger and M. Sprik, J. Phys. Chem. B 109, 6793 (2005).

${ }^{24}$ Y. Tateyama, J. Blumberger, M. Sprik, and I. Tavernelli, J. Chem. Phys. 122, 234505 (2005).

${ }^{25}$ J. Åqvist and T. Hansson, J. Phys. Chem. 100, 9512 (1996).

${ }^{26}$ S. Ten-no, F. Hirata, S. Kato, J. Chem. Phys. 100, 7443 (1994).

${ }^{27}$ H. Sato, F. Hirata, and S. Kato, J. Chem. Phys. 105, 1546 (1996).

${ }^{28}$ B. Roux, H. A. Yu, and M. Karplus, J. Phys. Chem. 94, 4683 (1990).
${ }^{29}$ D. Yokogawa, H. Sato, and S. Sakaki, J. Chem. Phys. 126, 244504 (2007).

${ }^{30}$ C. J. F Bottcher, Theory of Electric Polarization (Elsevier, Amsterdam, 1983).

${ }^{31}$ W. C. Still, A. Tempczyk, R. C. Hawley, and T. Hendrickson, J. Am. Chem. Soc. 112, 6127 (1990).

${ }^{32}$ J. Tomasi, B. Mennucci, and R. Cammi, Chem. Rev. 105, 2999 (2005).

${ }^{33}$ K. Naka, A. Morita, and S. Kato, J. Chem. Phys. 111, 481 (1999).

${ }^{34} \mathrm{M}$. Cossi and V. Barone, J. Chem. Phys. 112, 2427 (2000).

${ }^{35}$ J. W. Caldwell and P. A. Kollman, J. Phys. Chem. 99, 6208 (1995).

${ }^{36}$ S. E. Bradforth and P. Jungwirth, J. Phys. Chem. A 106, 1286 (2002).

${ }^{37}$ K. Ando, J. Chem. Phys. 115, 5228 (2001).

${ }^{38}$ R. W. Zwanzig, J. Chem. Phys. 22, 1420 (1954).

${ }^{39}$ H. Sato and S. Sakaki, J. Phys. Chem. A 108, 1629 (2004).

${ }^{40}$ H. J. Kim and J. T. Hynes, J. Chem. Phys. 93, 5194 (1990).

${ }^{41}$ M. A. Aguilar, J. Phys. Chem. A 105, 10393 (2001).

${ }^{42}$ H. J. C. Berendsen, J. P. M. Postma, W. F. van Gunsteren, and J. Hermans, Intermolecular Forces, edited by B. Pullman (Reidel, Dordrecht, 1981).

${ }^{43}$ S. W. Rick, S. J. Stuart, and B. J. Berne, J. Chem. Phys. 101, 6141 (1994).

${ }^{44}$ I. V. Leontyev and A. A. Stuchebrukhov, J. Chem. Theory Comput. 6, 1498 (2010).

${ }^{45}$ S. Lee and S. S. Park, J. Phys. Chem. B 115, 12571 (2011).

${ }^{46}$ S. J. Singer and D. Chandler, Mol. Phys. 55, 621 (1985).

${ }^{47}$ M. J. Frisch, G. W. Trucks, H. B. Schlegel et al., GaUSSIAN 03, Revision C.02, Gaussian, Inc., Wallingford, CT, 2004.

${ }^{48}$ M. W. Schmidt, K. K. Baldridge, J. A. Boatz, S. T. Elbert, M. S. Gordon, J. H. Jensen, S. Koseki, N. Matsunaga, K. A. Nguyen, S. Su, T. L. Windus, M. Dupuis, and J. A. Montgomery, J. Comput. Chem. 14, 1347 (1993).

${ }^{49}$ M. Kinoshita and F. Hirata, J. Chem. Phys. 106, 5202 (1997).

${ }^{50}$ B. M. Pettitt and P. J. Rossky, J. Chem. Phys. 84, 5836 (1986).

${ }^{51}$ Handbook of Chemistry and Physics, 78th ed., edited by D. R. Lide (CRC, Boca Raton, 1997).

${ }^{52}$ M. S. Banna, B. H. McQuaide, R. Malutzki, and V. Schmidt, J. Chem. Phys. 84, 4739 (1986).

${ }^{53}$ M. Faubel, Photoionization and Photodetachment, edited by C. Y. Ng (World Scientific, Singapore, 2000), Vol. 10A, Part 1, p. 634. 